\title{
A FORMAL COMPARISON OF METHODS PROPOSED FOR THE NUMERICAL SOLUTION OF FIRST KIND INTEGRAI EQUATIONS
}

\author{
R. S. ANDERSSEN AND P. M. PRENTER
}

(Received 7 August 1980)

(Revised 26 November 1980)

\begin{abstract}
A formal framework is constructed for the comparison of different stabilization techniques, such as Wiener filtering, regularization, Courant's method and LandweberStrand iterations, for the solution of first kind integral equations. It is shown that, when they are applied to convolution equations, all these methods can be reinterpreted as Wiener filters. This equivalence is then used to derive some specific results about regularization, Courant's method and Landweber-Strand iteration.
\end{abstract}

\section{Introduction}

For a comparison of the different methods proposed for some computational problem (such as the numerical solution of first kind integral equations), numerical experimentation with a representative set of test problems has proved quite useful in yielding qualitative results and conclusions about their performance. In part, such an approach was used by Graves and Prenter [10] in their study of the Landweber-Strand iteration for the solution of first kind Fredholm integral equations. Quantitatively, such assessment and classification is not possible without a formal framework for comparison. The aim of the present paper is to construct such a framework, and to use it to compare the Landweber-Strand iteration, Courant's method, regularization and Wiener filtering for the numerical solution of first kind integral equations.

CCopyright Australian Mathematical Society 1981 
Although the applicability of each of these methods is restricted to some subclass of first kind integral equations, they can all be applied to first kind convolution kernels. It is this fact which will be used to construct a formal framework of comparison. Initially, it will be necessary to define and develop relevant results for each of the methods to be examined. This will be done in Section 2. The formal framework of comparison will be constructed in Section 3 and used to define conditions under which the different methods are equivalent. In particular, conditions are identified under which the Landweber-Strand iteration can be interpreted as a Wiener filtering procedure.

\section{Notation and Preliminaries}

\subsection{The problem}

Formally, we are interested in the numerical solution of first kind convolution integral equations

$$
\mathbf{A} u=\int_{-\infty}^{\infty} A(t-\tau) u(\tau) d \tau=s(t), \quad-\infty<\tau, t<\infty
$$

In fact, we examine (2.1) under the following regularity assumptions:

(i) The kernel $A(t-\tau)$ is known explicitly and is such that A defines a linear filter which satisfies the following conditions:

(a) $\mathbf{A} \exp (i \omega t)=a(\omega) \exp (i \omega t)$, with $a(\omega)$, the transfer function of $\mathbf{A}$, independent of $t$;

(b) $a(\omega) \neq 0, \omega>0$.

The existence of the inverse filter $\mathbf{A}^{-1}=\mathbf{L}$, say, defined by $\mathbf{A}^{-1} \mathbf{A} \exp (i \omega t)=$ $\exp (i \omega t)$ is guaranteed by these conditions. Clearly,

$$
\mathbf{A}^{-1} \exp (i \omega t)=\mathbf{L} \exp (i \omega t)=l(\omega) \exp (i \omega t)
$$

with transfer function $l(\omega)=1 / a(\omega)$.

In addition, the adjoint of $\mathbf{A}^{*}$ of $\mathbf{A}$ will have transfer function $a^{*}(w)$, where $a^{*}(w)$ denotes the complex conjugate of $a(w)$; while rational function operators will have corresponding rational function transfer functions.

(ii) The signal $s(t)$ denotes a realization of a continuous mean-zero stationary stochastic process, and is only available as discrete observational data; namely

$$
\left\{d_{k}\right\}=\left\{d_{k}=s\left(t_{k}\right)+\varepsilon_{k}, k=0,1,2, \ldots, N-1, t_{k}=a+k \Delta, \Delta=\frac{b-a}{N}\right\},
$$

where the errors (noise) $\left\{\varepsilon_{k}\right\}$ denote realizations of a discrete mean-zero stationary stochastic process, and $[a, b]$ defines the interval on which the discrete 
data is collected. If the spectral density function associated with the signal $s(t)$ and the noise $\left\{\varepsilon_{k}\right\}$ are denoted by $g_{s}(\omega)$ and $g_{e}(\omega)$, respectively, then the spectral density function associated with the data $\left\{d_{k}\right\}$ will be defined by

$$
g_{d}(\omega)=g_{s}(\omega)+g_{e}(\omega) \text {. }
$$

With respect to the given data $\left\{d_{k}\right\}$, only estimates of $g_{d}(\omega)$ can be constructed numerically (for example via the smoothing of periodogram estimates-see Blocmfield [7]). If, however, appropriate assumptions cañ tee made auouit the smoothness of $s(t)$ relative to $\left\{\varepsilon_{j}\right\}$, the estimates of $g_{d}(\omega)$ can be used to infer qualitatively the nature of the decomposition defined by (2.3).

Thus, the problem becomes: with respect to given observational data $\left\{d_{k}\right\}$, construct numerically an approximation to the unknown solution $u(t)$ of $(2.1)$.

Other types of convolution integral equations could have been used instead of (2.1). For example,

$$
\int_{0}^{2 \pi} k(t-\tau) u(\tau) d \tau=s(t), \quad-\infty<t, \tau<\infty
$$

with

$$
k(t-\tau)=\sum_{j=1}^{\infty} a_{j} \cos (t-\tau), \quad a_{j} \neq 0, j=1,2, \ldots,
$$

since

$$
\int_{0}^{2 \pi} k(t-\tau)\left\{\begin{array}{c}
\sin j \tau \\
\cos j \tau
\end{array}\right\} d \tau=a_{j}\left\{\begin{array}{c}
\sin j t \\
\cos j t
\end{array}\right\}
$$

However, their use would not change the form of the results derived below. In addition, they can be viewed as special cases of (2.1).

\subsection{Wiener filtering}

The strategy developed by Anderssen and Bloomfield [2], [3] for the construction of Wiener filters for the numerical differentiation of given observational data $\left\{d_{j}\right\}$ extends naturally to the construction of Wiener filters for the solution of the first kind convolution integral equation (2.1) satisfying conditions (i) and (ii) of Section 2.1 (see Anderssen and de Hoog [4], Section 3). Formally, this reduces to the construction of Wiener filters for the application of $L=A^{-1}$ to the given observational data $\{d\}$. In particular, it can be shown that the implicit spectral differentiation formula (5.13) drived in Anderssen and Bloomfield [3] becomes

$$
u\left(t_{k} ; W(\omega)\right)=\sum_{-\pi<\omega,<\pi} \hat{W}\left(\omega_{j}\right) \exp \left(i \omega_{j} k\right) l\left(\omega_{j}\right) \tilde{d}_{j}, \quad k=0,1, \ldots, N-1,
$$


where

$$
\omega_{j}=2 \pi j / N, \quad \tilde{d}_{j}=\frac{1}{N} \sum_{k=0}^{N-1} d_{k} \exp \left(-i k \omega_{j}\right), \quad j=0,1, \ldots, N-1,
$$

denote the discrete frequencies and transforms, respectively, $l(\omega)=1 / a(\omega)$ denotes the transfer function of the inverse filter $A^{-1}$ corresponding to (2.1) (for example, $l(\omega)=i \omega$ when (2.1) corresponds to the differentiation formulation (2.1) of Anderssen and Bloomfield [3]), and $\hat{W}(\omega)$ denotes the window function chosen to approximate the optimal Wiener window function

$$
W(\omega)=g_{s}(\omega) /\left\{g_{s}(\omega)+g_{e}(\omega)\right\} \text {. }
$$

From the point of view of the subsequent discussion, the implicit formula characterizes the extension of Wiener filtering to the numerical solution of first kind convolution integral equations, since:

(i) When $s\left(t_{k}\right)=u\left(t_{k}\right)$ with $\mathbf{A}=\mathbf{I}$, the identity operator with transfer function $a(\omega) \equiv 1,(2.5)$ yields the standard Wiener formulas for filtering the signal $s(t)$ from given data $\left\{d_{k}\right\}$.

(ii) The term $\hat{W}(\omega)$ identifies the smoothing applied by the Wiener filtering, since the terms

$$
\exp \left(i \omega_{j} k\right) l\left(\omega_{j}\right) \hat{d}_{j}
$$

characterize the application of $\mathbf{L}=\mathbf{A}^{-1}$ to the frequency domain data $\left\{\exp \left(i \omega_{j} k\right) \tilde{d}_{j}\right\}$.

\subsection{Regularization}

For the operator equation (2.1), regularization can be defined formally as

$$
\min _{u \in \mathscr{I}}\left\{\|\mathbf{A} u-s\|_{2}^{2}+\alpha\|\mathbf{T} u\|_{2}^{2}\right\},
$$

where $\mathfrak{A}$ denotes some admissible class of solutions, $\mathbf{T}$ denotes a linear differential operator with constant coefficients, $\alpha$ denotes the regularization parameter, and $\|\cdot\|_{2}$ denotes the norm in $L_{2}[a, b]$-the Hilbert space of square integrable functions defined on the interval $[a, b]$. We assume that $\mathbf{T}$ is such that $\|\mathbf{T} u\|_{2}$ defines a norm. The more general situation where $\|\mathbf{T} u\|_{2}^{2}$ only defines a seminorm is not examined.

The Euler-Lagrange equations corresponding to (2.8) are given by

$$
\left(\mathbf{A}^{*} \mathbf{A}+\alpha \mathbf{T}^{*} \mathbf{T}\right) u_{\alpha}=\mathbf{A}^{*} s,
$$

where $u_{\alpha}$ is used to denote the regularization approximation's dependence on $\alpha$. Under the assumption that $A$ is densely invertible, (2.9) yields

$$
u_{\alpha}=\left(\mathbf{A}^{*} \mathbf{A}+\alpha \mathbf{T}^{*} \mathbf{T}\right)^{-1} \mathbf{A}^{*} \mathbf{A}\left(\mathbf{A}^{-1} s\right) \text {. }
$$


In addition, $\mathbf{T}$ is a linear filter which can be characterized as

$$
T \exp (i \omega t)=t(\omega) \exp (i \omega t), \quad t(\omega) \neq 0, \omega>0 .
$$

With respect to this form of regularization, an interpretation is possible which is similar to that given for Wiener filtering above. In fact, if we write

$$
s(t)=\sum_{-\pi<\omega_{j}<\pi} \tilde{d}_{j} \exp \left(i \omega_{j} t\right)
$$

then

$$
u_{\alpha}(t ; \mathbf{T})=\sum_{-\pi<\omega_{j}<\pi} \frac{1}{\left\{1+\alpha t^{*}\left(\omega_{j}\right) t\left(\omega_{j}\right) l^{*}\left(\omega_{j}\right) l\left(\omega_{j}\right)\right\}} \exp \left(i \omega_{j} t\right) l\left(\omega_{j}\right) \tilde{d}_{j},
$$

where $t^{*}(\omega)$ and $l^{*}(\omega)$ denote the complex conjugates of $t(\omega)$ and $l(\omega)$, yields a regularization counterpart of the implicit Wiener filtering formula (2.5).

This shows that the whole process of regularizing a convolution integral equation can be performed in the Fourier domain. As an immediate consequence, it follows that, for even gridded data, the number of operations required to evaluate the regularized solution $u_{\alpha}\left(t_{k}, \mathbf{T}\right), k=0,1,2, \ldots, N-1$ is $O(N \log N)$.

\subsection{Courant's method}

(See Courant and Hilbert [8], page 792.)

For the operator equation (2.1), one can formally define the point evaluation of its solution $u$ at $x$ by

$$
u(x)=\int_{-\infty}^{\infty} \delta(x-y) \mathbf{A}^{-1} s(y) d y .
$$

Under suitable regularity, the order of $\delta(x-y)$ and $\mathbf{A}^{-1}$ can be reversed to yield (see Allison [1], page 22)

$$
u(x)=\int_{-\infty}^{\infty}\left[\left(\mathbf{A}^{-1}\right)^{*} \delta(z)\right] s(x-z) d z
$$

where $\mathbf{A}^{*}$ denotes the adjoint of $\mathbf{A}$; but this form will not be examined here.

The essence of Courant's method is the replacement of the $\delta$-function in (2.13) by some well behaved parametrically defined $\delta$-shaped representation $\delta(x-y ; \alpha, \beta, \ldots, \gamma)$ with parameters $\alpha, \beta, \ldots, \gamma$. The approximate solution of $(2.1)$ is thereby reduced to the evaluation of

$$
u(x ; \alpha, \beta, \ldots, \gamma)=\int_{-\infty}^{\infty} \delta(x-y ; \alpha, \beta, \ldots, \gamma) \mathbf{A}^{-1} s(y) d y .
$$

An interpretation is possible which is similar to that given for Wiener filtering above, but it is necessary to observe that convolution with

$$
\delta(x-y ; \alpha, \beta, \ldots, \gamma)
$$


is equivalent to linear filtering with transfer function (see Koopmans [11], page 89)

$$
\hat{W}(\omega ; \alpha, \beta, \ldots, \gamma)=\int_{-\infty}^{\infty} \delta(x ; \alpha, \beta, \ldots, \gamma) \exp (-i \omega x) d x .
$$

Thus, on introducing the substitution (2.11) for $s(t)$ into (2.14), we obtain

$$
u(x ; \alpha, \beta, \ldots, \gamma)=\sum_{-\pi<\omega_{j}<\pi} \hat{W}(\omega ; \alpha, \beta, \ldots, \gamma) \exp \left(i \omega_{j} x\right) l\left(\omega_{j}\right) \tilde{d}_{j},
$$

which yields the implicit Wiener filtering counterpart for Courant's method.

\subsection{Landweber-Strand iteration}

(See Strand [15] and Graves and Prenter [10].)

The essence of this method is a generalization of Richardson's [14] iterative method for the solution of the matrix equation $A \mathbf{u}=\mathbf{s}$ (namely,

$$
\mathbf{u}^{(n+1)}=\mathbf{u}^{(n)}-\alpha_{n}\left(A \mathbf{u}^{(n)}-\mathrm{s}\right), \quad n=0,1,2, \ldots,
$$

where $u^{(0)}$ denotes the chosen starting solution, and the $\alpha_{1}, \alpha_{2}, \ldots$, are iteration parameters with $\alpha_{k}=\alpha_{k-P}(k>P)(P \equiv$ period of the iteration)) to the operator equation framework of (2.1) with period $P=1$ :

$$
u^{(n+1)}=u^{(n)}-\mathbf{D A}^{*}\left(\mathbf{A} u^{(n)}-s\right), \quad n=0,1,2, \ldots,
$$

where $u^{(0)}$ denotes the chosen starting solution and $\mathrm{D}=F\left(\mathrm{~A}^{*} \mathrm{~A}\right)$ with $F(t)$ chosen so that $t F(t)$ yields either a polynomial or rational function approximation to the Heaviside Unit Step function

$$
H_{\alpha}(t)= \begin{cases}1, & \alpha<t<1, \\ 0, & 0<t<\alpha .\end{cases}
$$

In essence, then, the Landweber-Strand iteration is optimized by choosing $t F(t)=H_{\alpha}(t)$. We therefore examine the consequences of this choice.

It follows from Graves and Prenter [10] that, with $u^{(0)} \equiv 0$,

$$
u^{(n+1)}=\left(\mathbf{I}-\left(\mathbf{I}-\mathbf{D A}^{*} \mathbf{A}\right)^{n+1}\right) \mathbf{A}^{-1} s .
$$

Thus, on introducing the substituion (2.11) for $s(t)$ into (2.18), we obtain $u^{(n+1)}(t ; F)$

$$
\begin{aligned}
& =\sum_{-\pi<\omega_{j}<\pi}\left[1-\left\{1-a^{*}\left(\omega_{j}\right) a\left(\omega_{j}\right) F\left(a^{*}\left(\omega_{j}\right) a\left(\omega_{j}\right)\right)\right\}^{n+1}\right] \exp \left(i \omega_{j} t\right) l\left(\omega_{j}\right) \tilde{d}_{j} \\
& =\sum_{-\pi<\omega_{j}<\pi}\left[1-\left\{1-F\left(1 / l^{*}\left(\omega_{j}\right) l\left(\omega_{j}\right)\right) / l^{*}\left(\omega_{j}\right) l\left(\omega_{j}\right)\right\}^{n+1}\right] \exp \left(i \omega_{j} t\right) l\left(\omega_{j}\right) \tilde{d}_{j},
\end{aligned}
$$

which yields the implicit Wiener filtering counterpart of Landweber-Strand iteration. For the interpretation and comparison of the smoothing term in (2.19), 
namely

$$
\begin{aligned}
1-\left\{1-a^{*}(\omega) a(\omega) F\left(a^{*}(\omega) a(\omega)\right)\right\}^{n+1} \\
=1-\left\{1-F\left(1 / l^{*}(\omega) l(\omega)\right) / l^{*}(\omega) l(\omega)\right\}^{n+1},
\end{aligned}
$$

with $\hat{W}(\omega)$ of $(2.5)$, the fact must be used that $a(\omega)$ corresponds to the eigenvalues of $A$ while $l(\omega)$ corresponds to the eigenvalues of $A^{-1}$. Thus, if $A^{-1}$ corresponds to $n$th order differentiation, then $|l(\omega)|$ is an increasing function of $\omega$ while $|a(\omega)|$ is a decreasing function. Since $t F(t)=H_{\alpha}(t)$ implies that

$$
F(1 / \tau) / \tau=H_{\alpha}^{0}(\tau)=1-H_{1 / \alpha}(t), \quad \tau=1 / t,
$$

it follows that, for this optimal choice,

$$
1-\left\{1-a^{*}(\omega) a(\omega) F\left(a^{*}(\omega) a(\omega)\right)\right\}^{n+1}=H_{\alpha}\left(a^{*}(\omega) a(\omega)\right)
$$

and

$$
1-\left\{1-F\left(1 / l^{*}(\omega) l(\omega)\right) / l^{*}(\omega) l(\omega)\right\}^{n+1}=H_{\alpha}^{0}\left(l^{*}(\omega) l(\omega)\right) .
$$

It is clear that the complicated nature of the filtering associated with Landweber-Strand iteration is a direct consequence of its iterative nature; and that a suboptimal choice for $t F(t)$ is one for which (2.21) is true asymptotically.

\section{Formal framework of comparison}

Using the results derived above, the following formal comparisons between Landweber-Strand iteration, Courant's method, regularization and Wiener filtering can be made. As is clear from the form of the results derived, the aim is to compare Landweber-Strand iteration, Courant's and regularization with Wiener filtering.

\subsection{Regularization and Wiener filtering}

The following result generalizes the equivalences derived by Anderssen and Bloomfield ([3]; Equations (6.16) and (9.6) and the second last quation on page 180).

THEOREM 3.1. For the convolution integral equation (2.1) and data $\left\{d_{k}\right\}$, which satisfy the conditions of Section 2.1, the formal equivalence of regularization and Wiener filtering defined by

$$
u\left(t_{k} ; \hat{W}(\omega)\right)=u_{\alpha}\left(t_{k} ; \mathrm{T}\right), \quad k=0,1,2, \ldots, N-1,
$$


holds when the smoothing $\hat{W}(\omega)$ which characterizes Wiener filtering is taken to be

$$
\lambda_{\alpha}(\omega ; t(\omega))=1 /\left\{1+\alpha t^{*}(\omega) t(\omega) l^{*}(\omega) l(\omega)\right\} .
$$

Proof. An immediate consequence of (2.5) and (2.12).

The importance of such an equivalence is the insight it gives about the nature of both Wiener filtering and regularization:

(i) The "stability" which regularization is known to exhibit numerically (see de Hoog [9] and Lukas [12]), because of the diagonal dominance it tends to impose on the matrix formulation resulting from the direct solution of $(2.1)$, is transferred to Wiener filtering.

(ii) The potential that Wiener filtering has for achieving a realistic separation of signal from noise, because of the statistical interpretation resulting from its stationary stochastic formulation, is transferred to regularization. It indicates that, unlike many methods of numerical analysis based on an abstract formalism, regularization does have the capacity to cope with the difficulties of non-exact data.

As well as the above trade-off in properties, this equivalence has two major consequences of direct practical utility. The first is the $O(N \log N)$ complexity result, which has been mentioned at the end of Section 2.3, while the second yields a strategy for determining a maximum-likelihood estimate for the regularization parameter $\alpha$.

The latter depends on utilizing the equivalence of Theorem 3.1 defined by $\hat{W}(\omega)=\lambda_{\alpha}(\omega ; t(\omega))$ to construct a parametric representation for the spectral density function of the data $g_{d}(\omega)$. In fact, if we assume that the spectrum of the errors, $g_{e}(\omega)$, was known up to a constant, namely

$$
g_{e}(\omega)=b h(\omega)
$$

with $h(\omega)$ known (for example, for truly uncorrelated errors $h(\omega) \equiv 1$ ), and the spectrum for the signal, $g_{s}(\omega)$, can be modelled as (see (2.7))

$$
g_{s}(\omega)=b h(\omega) /\left\{\alpha t^{*}(\omega) t(\omega) l^{*}(\omega) l(\omega)\right\},
$$

then $\hat{W}(\omega)=\lambda_{\alpha}(\omega ; t(\omega))$ and

$$
g_{d}(\omega)=b h(\omega)\left[1+1 /\left\{\alpha t^{*}(\omega) t(\omega) l^{*}(\omega) l(\omega)\right\}\right]
$$

yields a parametric representation for $g_{d}(\omega)$ consistent with the equivalence.

Thus, as defined by (3.5), the spectrum of the observed process $\left\{d_{k}\right\}$ depends on the parameter of interest, $\alpha$, and an overall scale parameter, $b$. But, since the distribution of a process is determined by its spectrum, at least for Gaussian models, it follows that $\alpha$ may be estimated from the statistical properties of the 
observations $\left\{d_{j}\right\}$. In fact, we have

THEOREM 3.2. The maximum likelihood estimate of $\alpha$ is determined as that value of $\alpha$ which minimizes the following approximation for the likelihood of $\alpha$

$$
\begin{array}{r}
\frac{N+1}{2} \log \left(\sum_{-\pi<\omega_{j}<\pi} I\left(\omega_{j}\right) /\left[1+1 /\left\{\alpha t^{*}\left(\omega_{j}\right) t\left(\omega_{j}\right) l^{*}\left(\omega_{j}\right) l\left(\omega_{j}\right)\right\}\right]\right) \\
+\frac{1}{2} \sum_{-\pi<\omega_{j}<\pi} \log \left[1+1 /\left\{\alpha t^{*}\left(\omega_{j}\right) t\left(\omega_{j}\right) l^{*}\left(\omega_{j}\right) l\left(\omega_{j}\right)\right\}\right]
\end{array}
$$

where

$$
\begin{aligned}
I\left(\omega_{j}\right) & =\frac{1}{2 \pi N}\left|\sum_{k=0}^{N} d_{k} \exp \left(i k \omega_{j}\right)\right|^{2} \\
& =N\left|\tilde{d_{j}}\right|^{2} / 2 \pi
\end{aligned}
$$

denotes the periodogram of the data; and, in the second sum, the infinite term at $\omega_{j}=0$ is replaced by an arbitrary quantity, which is usually taken to be zero.

Proof. It follows that given by Anderssen and Bloomfield [2].

When implementing an algorithm based on this equivalence, it is not only necessary to find the $\alpha$ which minimizes (3.6), but also necessary to choose $T$ (and thus $t(\omega)$ ), since the definition of (3.6) involves $t(\omega)$ as well as $l(\omega)$. The procedure developed by Anderssen and Bloomfield [2], [3] reduces to choosing the $\mathbf{T}$ from some appropriate and previously specified class so that, with respect to the associated value of $\alpha$ which minimizes (3.6), the resulting model of $g_{d}(\omega)$ defined by (3.5) yields a reasonable fit to the periodogram $I\left(\omega_{j}\right)$ of the data $\left.; d_{k}\right\}$ as defined by (3.7). Alternative methods for choosing $\mathbf{T}$ and $\alpha$ have been proposed and examined in some detail by Wahba [16], [17]. These methods are based on the use of generalized cross validation. Most of the discussion of regularization in the literature centers on finding $\alpha$ assuming that $T$ has been given in advance. Lukas [13], however, has shown that, if best results are to be obtained, it is necessary to examine optimality criteria for approximations as a function of both $\mathbf{T}$ and $\alpha$. In addition, he has found that the choice of $\mathbf{T}$ is the more crucial step and has developed a procedure for determining $T$ before $\alpha$.

\subsection{Wiener filtering, regularization and Landweber-Strand iteration}

As an automatic consequence of (2.12) and (2.20), we have

TheOREM 3.3. For convolution integral equations (2.1) and data $\left\{d_{k}\right\}$, which satisfies the conditions of Section 2.1, the formal equivalence of regularization and 
Landweber-Strand iteration defined by

$$
u_{\alpha}\left(t_{k} ; \mathrm{T}\right)=u^{(n)}\left(t_{k} ; F\right), \quad k=0,1,2, \ldots, N-1,
$$

holds when the linear filter $\mathbf{T}$ of regularization and the function $F$ of LandweberStrand iteration are related by

$$
1 /\left\{1+\alpha t^{*}(\omega) t(\omega) l^{*}(\omega) l(\omega)\right\}=1-\left[1-F\left(1 / l^{*}(\omega) l(\omega)\right) / l^{*}(\omega) l(\omega)\right]^{n} .
$$

On invoking the equivalence of regularization and Wiener filtering of Theorem 3.1, it follows that the role played by the right-hand side of (3.9) is equivalent to that of the smoothing of the Wiener filter defined by $\hat{W}(\omega)$. But, it follows from (2.21) that, in the limit, the Landweber-Strand iteration only yields a form for the right-hand side of (3.9) which corresponds to the low pass filtering of all frequencies such that

$$
F\left(1 / l^{*}\left(\omega_{j}\right) l\left(\omega_{j}\right)\right) / l^{*}\left(\omega_{j}\right) l\left(\omega_{j}\right) \leqslant \frac{1}{\alpha} .
$$

However, from a Wiener filtering point of view, low pass filtering is not optimal. In addition, since, with respect to a given $n$, the optimal $\alpha \rightarrow 0$ as $n \rightarrow \infty$, this shows that all frequencies are passed in the limit. This interpretation of Landweber-Strand iteration also yields an explanation of why the methods investigated by Graves and Prenter [10], when $t F(t)$ is taken to be a polynomial approximation to $H_{\alpha}(t)$, tend to yield rather slow convergence. Namely, the range of a polynomial approximation to $H_{\alpha}(t)$ can extend below zero and above 1 , thereby resulting in the negative enhancement at certain of the higher frequencies, and overenhancement at certain of the lower.

It also follows from (3.9) that both $n$ and $F$ determine the smoothing properties of Landweber-Strand iteration. The various forms that the right-hand side of (3.9) can take represent a trade-off between $n$ and $F$. If it is assumed that $n$ is specified in advance (there are various ways in which this can be done; see Wahba [17]), then (3.9) can be used to define an appropriate form for $F$; namely,

$$
F\left(1 / l^{*}(\omega) l(\omega)\right) l^{*}(\omega) l(\omega)=1-\left[1-\lambda_{\alpha}(\omega ; t(\omega))\right]^{1 / n} .
$$

Thus, with respect to a chosen form for $\lambda_{\alpha}(\omega ; t(\omega))$ derived from a Wiener filtering-regularization context, the right-hand side of (2.12) defines the form $F(1 / \tau) / \tau$ should take if after $n$ steps of the Landweber-Strand iteration, the filtering is to correspond to that defined by $\lambda_{\alpha}(\omega ; t(\omega))$. Further, since the right-hand side of (3.11) has the same basic shape as $\lambda_{a}(\omega ; t(\omega))$ itself, it follows that if an approximation to the right-hand side of (3.11) is sought, then it should take the form of a truncated continued fraction. Independently, Bierbaum [6] has shown that, when compared with those of Graves and Prenter [10], better 
results are obtained from the Landweber-Strand iteration when $t F(t)$ is constructed to be a truncated continued fraction approximation to $H_{\alpha}(t)$.

\subsection{Courant's method, regularization and Wiener filtering}

As indicated in Section 2.4, Courant's method can be interpreted as an implicit Wiener filter and thereby yields a natural framework for its generalization to non-convolution equations. A number of authors have rediscovered and applied Courant's method in various forms (see Backus and Gilbert [5]), but these will not be pursued here.

When Courant's method is modelled in the form (2.13), its obvious equivalence with Wiener filtering leads naturally via Theorem 3.1 to its equivalence with regularization which reduces to choosing

$$
\hat{W}(\omega ; \alpha, \beta, \ldots, \gamma)=1 /\left\{1+\alpha t^{*}(\omega) t(\omega) l^{*}(\omega) l(\omega)\right\} .
$$

Allison [1] has asserted without a rigorous proof that on replacing the $\delta$-function in (2.13) by some well behaved parametrically defined $\delta$-shaped representation $\delta(x-y ; \alpha, \beta, \ldots, \gamma)$, Courant's method reduces to the form

$$
u(x)=\mathbf{A}_{\alpha}^{-1} s
$$

where $\mathbf{A}_{\alpha}^{-1}$ takes the form

$$
\mathbf{S}_{\alpha} \mathbf{A}^{-1}
$$

with $\mathbf{S}_{\alpha}$ corresponding to some smoothing operator (of ten called a regularizator of $\mathbf{A}$ ); and then argued that one thereby obtains an identification with regularization on writing

$$
\mathbf{S}_{\alpha}=\mathbf{A}^{*}\left(\mathbf{A A}^{*}+\alpha \mathbf{I}\right)^{-1} \mathbf{A}
$$

But, this does not yield an explicit characterization of the equivalence of these two methods.

The framework of Sections 2.1-2.5 can be used to establish a sense in which such operators $\mathbf{S}_{\alpha}$ exist, and to define the explicit nature of the equivalence between Courant's method, Wiener filtering and regularization for convolution operators. Now, from (2.16), it follows that

$$
u(t ; \alpha, \beta, \ldots, \alpha)=\sum_{-\pi<\omega_{j}<\pi} \hat{W}\left(\omega_{j} ; \alpha, \beta, \ldots, \gamma\right) \exp \left(i \omega_{j} t\right) l\left(\omega_{j}\right) \tilde{d}_{j} .
$$

Thus, it follows from the Wiener filter interpretation of $\hat{W}(\omega)$ in Section 2.2, (ii), that the transfer function $\hat{W}\left(\omega_{j} ; \alpha, \beta, \ldots, \gamma\right)$, defined by (2.15), defines a smoothing operator (linear filter) applied to $A^{-1} s$. In fact, it follows from Theorem 3.1 that if $\delta(x-y ; \alpha, \beta, \ldots, \gamma)$ is chosen so that the transfer function 
it generates via (2.15) satisfies

$$
\hat{W}(\omega ; \alpha, \beta, \ldots, \gamma)=\lambda_{\alpha}(\omega ; t(\omega))
$$

then the smoothing operator $\mathbf{S}_{\alpha}$ corresponds to the regularization operator of (2.10)

$$
\left(A^{*} \mathbf{A}+\alpha T^{*} T\right)^{-1} \mathbf{A}^{*} \mathbf{A}
$$

In this way, we have established

THEOREM 3.4. For convolution integral equation (2.1) and data $\left\{d_{k}\right\}$, which satisfy the conditions of Section 2.1, the formal equivalence of Courant's method with Wiener filtering and regularization defined by

$$
u\left(t_{k} ; \alpha, \beta, \ldots, \gamma\right)=u\left(t_{k} ; \hat{W}(\omega)\right)=u_{\alpha}\left(t_{k} ; \mathbf{T}\right), \quad k=0,1,2, \ldots, N-1,
$$

holds when $\delta(x-y ; \alpha, \beta, \ldots, \gamma)$ is chosen so that the transfer function it generates via (2.15) satisfies (3.16).

\section{References}

[1] H. Allison, "Inverse unstable problems and some of their applications", The Math. Scientist 4 (1979), 9-30.

[2] R. S. Anderssen and P. Bloomfield, "A time series approach to numerical differentiation", Technometrics 16 (1974), 69-75.

[3] R. S. Anderssen and P. Bloomfield, "Numerical differentiation procedures for non-exact data", Numer. Math. 22 (1974), 157-182.

[4] R. S. Anderssen and F. R. de Hoog, "Application and numerical solution of Abel-type integral equations", Proceedings of the International Conference on "Improperly Posed Problems: Theory and Practice" (1980) (to appear).

[5] G. E. Backus and F. J. Gilbert, "Numerical application of a formalism for geophysical inverse problems", Geophys. J. R. astr. Soc., 13 (1967), 247-276.

[6] R. Bierbaum, A continued fraction iteration for ill-posed equations (M. Sc. Thesis, Mathematics, Colorado State University, Fort Collins, in preparation, 1980).

[7] P. Bloomfield, Fourier analysis of time series: an introduction (Wiley Series in Probability and Mathematical Statistics, Wiley, New York, 1976).

[8] R. Courant and D. Hilbert, Methods of mathematical physics (Interscience Publishers, Inc., New York, 1953).

[9] F. R. de Hoog, "Review of Fredholm equations of the first kind" The application and numerical solution of integral equations (eds. R. S. Anderssen, F. R. de Hoog and M. A. Lukas) (Sijthoff and Noordhoff, 1980), 119-134.

[10] J. Graves and P. M. Prenter, "Numerical iterative filters applied to first kind Fredholm integral equations", Numer. Math. 30 (1978), 281-299.

[11] L. H. Koopmans, The spectral analysis of time series (Academic Press, New York and London, 1974). 
[12] M. A. Lukas, "Regularization", in The application and numerical solution of integral equations (eds. R. S. Anderssen, F. R. de Hoog and M. A. Lukas) (Sijthoff and Noordhoff, 1980), $151-182$.

[13] M. A. Lukas, "A practical method for the application of regularization to first kind equations with noisy data" (in preparation).

[14] L. F. Richardson, "The approximate arithmetical solution by finite differences of physical problems involving differential equations with an application to the stress in a masonry dam", Phil. Trans. Roy. Soc. London, A.210 (1910), 307-357.

[15] 0 . N. Strand, "Theory and methods related to the singular function expansion and Landweber's iteration for integral equations of the first kind", SIAM J. Numerical Anal. 11 (1974), 798-825.

[16] G. Wahba, "Practical approximate solutions of linear operator equations when the data are noisy", SIAM J. Numerical Anal. 14 (1977), 651-667.

[17] G. Wahba, "Ill-posed problems: numerical and statistical methods for mildly, moderately and severely ill-posed problems with noisy data", University of Wisconsin-Madison, Statistics Department Technical Report No. 595 (1980).

Division of Mathematics and Statistics, C.S.I.R.O.

P.O. Box 1965

Canberra City

A.C.T. 2601

and

Colorado State University

Department of Mathematics

Fort Collins

Colorado 80523

U.S.A. 